\title{
ROBERT MUSIL:
}

LA CIENCIA Y LA VIDA

Juan Cristóbal Cruz*

Nuestra ciencia, es decir, el producto más característico, la gloria más cierta y la más personal de nuestro espíritu.

Paul Valéry ${ }^{1}$

En el mundo intelectual vienés de la primera mitad del siglo XX, una de las discusiones más significativas se producirá en torno a las implicaciones sociales y culturales de la ciencia. Naturalmente en los círculos técnicos y científicos se tiene una gran esperanza en lo que ella puede significar como modelo general de saber, y este sentimiento se acompaña de una efervescencia de concepciones, como lo narra Karl Popper:" "Después del derrumbe del Imperio Austríaco se había producido una revolución en Austria: el aire estaba cargado de lemas e ideas revolucionarias, y de nuevas y a menudo audaces teorías." "Este género de expectativas es fomentado por la influencia aún poderosa de las grandes personalidades de fines del siglo XIX, como la del físico y filósofo Ludwig Boltzmann (18441906) -seguido, entre otros, por Niels Bohr-pero en particular la de

* Licenciado en Derecho, ITAM y Doctor en Ciencias Políticas, La Sorbona.

${ }^{1}$ Paul Valéry, Mais qui est donc Européen?, La crise de l'esprit en Variété, 1967, París, Gallimard, vol. 1, p. 50.

${ }^{2}$ Karl Popper, Conjeturas y refutaciones, El desarrollo del conocimiento científico, 1994, Barcelona, Paidós, p. 58.

${ }^{3}$ Ibid. 
JUAN CRISTÓBAL CRUZ

Ernst Mach (1838-1916). Para tener una idea de su enorme ascendencia basta recordar los siguientes reconocimientos explícitos o implícitos: en su honor se creará la Asociación Ernst-Mach que dará pie al Círculo de Viena; también con su nombre se bautizará la velocidad del sonido; ${ }^{4}$ por su parte el filósofo norteamericano William James, luego de tratarlo en Praga en 1882, comentará no haber conocido a nadie que le "haya provocado tan fuerte impresión de puro genio intelectual", 5 en tanto que, más tarde, Albert Einstein reconocerá la profunda influencia que ejerció Mach en su juventud, ${ }^{6}$ y el mismo Lenin se sentirá obligado a atacarlo con un panfleto. ${ }^{7}$

Estas influencias y la atmósfera cultural que propician, permiten entender por qué los miembros del Círculo de Viena ven en la explicación científica la forma 'primordial de cognición'8 y piensan que la filosofía debe imitar el camino seguro de la ciencia. ${ }^{9}$ En el entorno cercano al Círculo de Viena, Robert Musil -escritor, ingeniero e inventor del Variationskreisel (instrumento de discos giratorios polícromos para medir la percepción de los colores)- coincide con el joven Karl Popper y con el polifacético Otto Neurath ${ }^{10}$ en su convicción de que

${ }^{4}$ Técnicamente se denomina así la relación entre la velocidad del flujo del aire y la velocidad del sonido. William Johnston, 1985, París, PUF, p. 222.

${ }^{5}$ Citado por Philipp Frank, en Between Physics and Philosophy, 1941, Cambridge, Mass., p. 211.

${ }^{6}$ Albert Einstein, "Autobiographical Notes", en P. A. Schlipp (editor), Albert Einstein: Philosopher-Scientist, vol. I, p. 21.

${ }^{7}$ Lenin, Materialismo e empirocriticismo, 1908.

${ }^{8}$ Herbert Feigl y Stephen Toulmain, Legado del Positivismo Lógico, 1981, Teorema, p. 18.

${ }^{9}$ Ibid., p. 48.

${ }^{10}$ La atmósfera vienesa aún favorecía la presencia de personalidades universales como Musil o Neurath. El caso de Neurath es significativo y sorprendente, no sólo es uno de los miembros eminentes del Círculo de Viena sino, como lo subraya William M. Johnston, también realiza "investigaciones originales en física, en matemáticas, en lógica, en historia de la literatura alemana, en arquitectura, en economía, en sociología, en historia antigua, en teoría política y en geografía"( L'esprit viennois, p. 237). En cuanto a los 
ROBERT MUSIL

el esclarecimiento de los problemas epistemológicos puede tener efectos no sólo en el campo científico, sino también en la ética y 'hasta en la política'.

Sin embargo, esta disposición no es compartida en amplios sectores de la sociedad vienesa de la época. En particular en los medios filosóficos y literarios predomina el escepticismo ante la importancia paradigmática del saber científico y la desconfianza frente a su significado cultural. Incluso el interés que suscitan los trabajos de Mach o de su discípulo Fritz Mauthner en estos círculos, proviene del deseo de refutarlos. Mauthner mismo ilustra la incomprensión entre ambos sectores cuando, luego de publicar sus Contribuciones a una critica del lenguaje (editada en tres volúmenes entre 1901 y 1902) y creyendo encontrar un feliz eco de su obra en la Carta de Lord Chandos (1902), escribe a Hofmannsthal para expresarle su agradecimiento. El filósofo no se da cuenta de que en la obra del poeta no se busca acabar-como lo quiere su posición nominalista- con esa barrera al verdadero acceso al conocimiento que es la vieja e ingenua confianza (metafísica) en las palabras. En realidad Hofmannsthal pretende evidenciar la catástrofe que representa el fin de las ilusiones que la tradición occidental había depositado en el lenguaje. ${ }^{12}$ El malestar por los efectos culturales de la ciencia se hace patente, por ejemplo, cuando en sus Cartas de un viajero a su regreso, escritas en el transcurso de 1907, el mismo Hugo von Hofmannsthal se lamenta de que debido a la acción de las ciencias la visión experimentada por Rama Krishna al momento de su iluminación quiera reducirse a una simple 'anomalía del sistema nervioso', como lo afirma un religioso inglés. ${ }^{13}$ Ante el escándalo de contactos que Musil tiene con el Círculo de Viena, no se cuenta con mucha información; pero sabemos que frecuenta la casa y el medio de Ludwig von Mises. Véase Jean-Pierre Cometti, Le philosophe et la poule de Kircher, p. 27.

${ }^{11}$ Karl R. Popper, op. cit., p. 25.

12 J. Le Rider, Hugo von Hofmmannsthal, Historicisme et modernité, 1995, París, PUF, p. 78-9.

${ }^{13}$ H. Hofmannsthal, Lettres du voyageur à son retour, 1992, París, Gallimard, p. 157. 
"inferir el complejo de pasión, creación poética e intelectual que llamamos la vida de Goethe, de la estructura de su cerebro", 14 por distintos caminos - pero bajo la misma ascendencia postkantiana- la Filosofia de la vida, como la filosofia del primer Wittgenstein, aspiran a demostrar que el conocimiento científico sólo es válido para un ámbito determinado del saber y no precisamente el fundamental.

Paradójicamente, en estos mismos años y en el contexto de la Europa central, es en Alemania, en el pueblo científica y tecnológicamente más inventivo, como lo subraya Arnold Gehlen, ${ }^{15}$ y que gusta de identificarse con esa representación del deseo de conocimiento absoluto que es el mito fáustico, donde fermenta el mayor malestar frente al desarrollo de la ciencia y de la técnica. En efecto, en las aulas de la Universidad de Munich, Max Weber diagnostica que se prefigura como consecuencia del 'mundo de la razón' un horizonte desencantado y una prisión de hierro carente de contenido espiritual. Al mismo tiempo, en Estrasburgo Georg Simmel observa la creciente incapacidad de la vida subjetiva para hacer frente a la perfección y a la complejidad del nuevo ámbito objetivo, ${ }^{16}$ situación que despoja al individuo de una visión sintética de la realidad. Haciendo eco a Simmel, Musil en su novela El hombre sin atributos resume esta situación: "Estos últimos cien años nos han permitido acrecentar significativamente el conocimiento de nosotros mismos, de la naturaleza y de las cosas en general; pero de ello resulta que todo el orden que ganamos en los detalles lo perdemos en el conjunto, de manera que disponemos cada vez de más órdenes (en plural), y cada vez de menos orden (en singular). " ${ }^{17}$ Este

${ }^{14}$ W. Dilthey, "Las ciencias del espíritu son un todo independiente junto a las ciencias de la naturaleza", Ensayistas alemanes, 1995, México, Cien del mundo, p. 280.

${ }^{15}$ Arnold Gehlen, Anthoropologie et psychologie sociale, 1986, París, PUF.

${ }^{16}$ Por ejemplo, Georg Simmel, De la esencia de la cultura en El individuo y la libertad, 1986, Península, p. 126-7.

${ }^{17}$ Musil, Der Mann ohne Eigenschaften, vol I, Gesammelte Werke, 1978, Hamburgo, Rowohlt, 9 vols., p. 379. 
ROBERT MUSIL

estado de cosas explica que haya quienes, como Max Scheler, crean urgente demandar que la ciencia se subordine a una visión cultural más alta. Pero la crítica más elaborada e influyente y que no puede ser ignorada para entender adecuadamente esta época es la del filósofo de la Selva Negra, Martin Heidegger.

\section{La ciencia y de la técnica como olvido}

La reflexión en torno a la ciencia y a la técnica no es secundaria en el pensamiento y en la obra de Heidegger. Bajo influencia del célebre escritor Ernst Jünger, sobre todo de su libro El trabajador, ${ }^{18}$ Heidegger considera que el problema fundamental de la época contemporánea reside en particular en el 'estilo técnico de las ciencias modernas' 19 Esta interpretación será más clara en los trabajos posteriores a $E l$ ser $y$ el tiempo, ${ }^{20}$ donde ciencia y técnica serán señaladas como las expresiones más representativas y acabadas de la metafísica de la modernidad, al grado de llamarlas 'metafísica finalizada'. Esto significa que a pesar de la neutralidad normativa reivindicada por el pensamiento de Heidegger, detrás de sus afirmaciones hay un juicio negativo respecto a la época. ${ }^{21}$ Es decir, la ciencia y la técnica deben ser consideradas

${ }^{18}$ Ernst Jünger, Der Arbeiter. Herrschaft und Gestalt, 1932.

${ }^{19}$ Heidegger, Nietzsche, 1971, París, Gallimard, p. 213.

${ }^{20}$ Como se ha señalado, el problema de la ciencia, de la técnica y la de subjetividad en general aún son problemáticas y ambiguas en su obra mayor. Véase, Dreyfus, L. Hubert, "History of the Being of Equipment”, Heidegger: A critical Reader, 1993, Estados Unidos, Blackwell, Cambridge.

${ }^{21}$ En diferentes trabajos, Luc Ferry y Alain Renaut, han mostrado la ambigüedad constante de Heidegger a este respecto. Incluso en El ser y el tiempo, que pretende ser un trabajo estrictamente fenomenológico, el uso del lenguaje y el tipo de oposiciones evocan no sólo una visión moral sino incluso una heroica del Dasein. Pierre Bourdieu (L'ontolgie Politique de Martin Heidegger) hace una crítica análoga al mostrar cómo Heidegger incurre constantemente en la práctica de la 'denegación'. Cuando en cada enunciado que implica una 


\section{JUAN CRISTÓBAL CRUZ}

como la culminación del proceso moderno de decadencia respecto a la comprensión del Ser y de su Verdad. Para entender esta crítica del mundo moderno, es necesario situarla en la lectura de la historia de la metafísica occidental que hace este filósofo.

Para los griegos, defiende Heidegger, tal y como lo muestra en particular el caso de los filósofos presocráticos, la naturaleza (physis) tenía una significación radicalmente distinta a la que le otorga el hombre moderno. Era entendida como el sinónimo del ente en su totalidad, es decir, como el primer y esencial nombre de los seres en sí mismos y en su unidad, en tanto que sus fenómenos - 'lo que sale a la luz del día'22 eran interpretados como el despliegue espontáneo y sin coacción de sí misma. Sin embargo, esta forma de mostrarse no implicaba una transparencia plena de la naturaleza a la mirada del hombre, ya que para los griegos los fenómenos se presentaban y se mostraban a la luz, al mismo tiempo que se replegaban y permanecían bajo la oscuridad y el misterio ("en el de-velamiento mora el ocultamiento y la custodia"23), a la manera de la experiencia estética del templo griego en la que el visitante percibe la tensión entre la presencia exterior y algo que la excede y se resiste a mostrarse, se oculta. Según Heidegger, esta comprensión evitaba una visión antropocentrista de los fenómenos -ya que el 'llevar a lo luminoso' era, al mismo tiempo, un mantener al hombre frente al Misterio- y permitía reconocer la capacidad de poiesis (creación) de la naturaleza. A diferencia de lo que sucederá en el mundo moderno, esta idea de creación derivaba de la noción de lo que es producido o formado a partir de un modelo o de un saber dirigido (techné). La

marca de la subjetividad, niega que así sea: por ejemplo cuando utiliza la noción de autenticidad niega que se trate "de una crítica moralizante del Dasein" o cuando habla de decadencia del Ser inmediatamente señala que debe pensarse no a partir del hombre sino a partir del Ser (El ser y el tiempo, p. 86-7). Luc Ferry y Alain Renaut, Sistème et critique, Essais sur la critique de la raison dans la philosophie contemporaine, 1992, Bruxelles, Ousia.

${ }^{22}$ El concepto de fenómeno, §7, El ser y el tiempo, 1974, México, FCE, p. 39.

${ }^{23}$ Heidegger citado por George Steiner, Heidegger, 1986, México, FCE, p. 176. 
ROBERT MUSIL

techné como la poiesis eran entendidas como un descubrir y llevar a su verdadero ser lo que ya es inherente a la physis (es decir, como "el acto de hacer pasar una cosa del no ser al ser", como lo indicaba Platón ${ }^{24}$ ) en el sentido ya mencionado, y que debe ser subrayado, de un 'mostrar-ocultando'.

Heidegger considera que ya en la misma noción aristotélica de substancia y presencia constante -complementado con la búsqueda de 'causas primeras' y de 'principios dinámicos'- se encuentran en germen los fundamentos de la interpretación moderna del Ser. En efecto, la categoría de substancia atraviesa la Edad Media, y con el pensamiento cartesiano adquirirá un rasgo más preponderante aún. En éste, con la distinción entre substancia extensa y substancia pensante o espiritual, se concibe lo existente a partir del sujeto, "el ser del ente como presencia para un sujeto (de la representación)..." ${ }^{25}$ con el propósito de facilitar, como lo afirma explícitamente Descartes, las bases intelectuales que permitan hacer del hombre el 'señor de la naturaleza'. En esta voluntad de nous rendre comme maîtres et possesseurs de la nature ${ }^{26}$ proclamada por Descartes se revela, según Heidegger, el carácter de falsificación y decadencia de la interpretación moderna del Ser, ya que con ella desaparece toda noción de misterio y trascendencia y, de ahora en adelante, lo existente debe interpretarse y reducirse sólo a aquello que el Sujeto se representa. De esta forma el Mundo no puede sino convertirse en una simple imagen o espejo del hombre, centro de referencia del ente en su totalidad.

La tendencia implícita en la dualidad metafísica cartesiana sujetoobjeto que ha facilitado el advenimiento de la visión científica del mundo, se ha radicalizado en la actual época metafísica de la técnica. ${ }^{27}$

${ }^{24}$ Véase Cornelius Castoriadis, Les carrefours du labyrinthe, 1978, París, Seuil, t. I. p. 293. En este sentido el arte es imitación para los griegos.

25 "La cuestión de la técnica", Essais et conférences, 1958, Gallimard, París.

${ }^{26}$ Descartes, Discours de la méthode, 1990, París, Agora, p. 132.

${ }^{27}$ En su interesante artículo History of the Being of Equipment (op. cit.), Hubert Dreyfus defiende la idea que la concepción implícita en Ser y tiempo 


\section{JUAN CRISTÓBAL CRUZ}

La pretensión de determinar desde sí la totalidad, es entendida por Heidegger como la esencia de toda relación del hombre con los entes en la época moderna, como lo dice en El ser y el tiempo: "El bosque es parque forestal, la montaña cantera, el río fuerza hidráulica, el viento es viento 'en las velas'." 28 Por lo mismo, la ciencia no es sino una manifestación más de esa concepción metafísica fundamental. La crítica se sustenta en una argumentación que conviene mencionar rápidamente.

\section{Pensar la ciencia desde la técnica}

Heidegger afirma que la esencia de la ciencia reside en la investigación, por la que el conocimiento se convierte en un procedimiento en un aspecto del ente: por ejemplo, en Galileo que deja de lado las preguntas por las causas del movimiento o del reposo, para sólo interesarse en el cómo de la aceleración. ${ }^{29}$ A su vez este procedimiento exige rigor y exactitud en su ámbito específico, cada experiencia (es decir la reproducción controlada de efectos) busca reducir los fenómenos a datos y objetos calculables. Pero lo que se hace con cada parcela del saber se hace igualmente con la naturaleza en su totalidad, reduciéndola, a fin de cuentas, a un lenguaje matemático, tal y como lo proclamaba el mismo Galileo.

Pero para Heidegger la investigación no sólo es la manipulación de cosas tales como el átomo o los genes, sino ante todo una forma de organización social. En efecto, la investigación supone una labor inscrita en un marco institucional y productora de un tipo específico de hombre: el investigador. La ciencia y el conocimiento por ella producido-aun

representa el paso decisivo a la visión tecnológica del mundo. Por nuestra parte, siguiendo con reservas a Rorty en este punto, creemos que Heidegger y Dreyfus conceden una importancia exagerada e ilusoria al discurso filosófico dentro de la cultura. La filosofía es sólo un discurso explicativo entre otros: por ejemplo, el literario y el mismo discurso científico.

${ }^{28}$ M. Heidegger, El ser y el tiempo, op. cit., p. 84.

${ }^{29}$ Ilya Prigogine e Isabelle Stenger, La nouvelle alliance, 1979, Seuil, p. 99. 
en el campo de las llamadas ciencias históricas o del espíritu-se revelarían como técnica. De esta forma, Heidegger pretende mostrar que la técnica no es un fenómeno marginal (una simple derivación aplicada de la ciencia) o una característica más de la modernidad (una actividad social entre otras), sino que ella constituye su dinámica central, su metafísica.

Si este desarrollo histórico es tácitamente trágico, se debe a que la técnica no sólo expresa una voluntad de instrumentalizar al Ser, sino también y sobre todo a que conlleva (como destino ineluctable) el olvido del Ser. La época de la técnica olvida que el Ser es su propio substrato (visto de esta forma, nuestra época es un incesante preguntar vano, puesto que ha olvidado las preguntas fundamentales; la esencia misma de la ciencia reside en que su forma de preguntar es incapaz de preguntar por sus propios fundamentos, razón por la cual Heidegger afirmaba que 'la ciencia no piensa'). Peor, no sólo es un olvido sino una violencia ejercida por el hombre: "La desocultación que rige la técnica moderna se da bajo la forma de la 'provocación' (Herausfordern) a través de la cual la naturaleza es obligada a ceder una energía que como tal, pueda ser extraída (herausgefördert) y acumulada." 30

La misión del filósofo debe consistir, precisamente, en recordarnos que la técnica moderna es ella misma, al fin de cuentas, una expresión del Ser, una manifestación más de las 'desocultaciones' (históricas) del Ser, pero esta vez bajo la forma de Gestell ('enmascaramiento', 'armadura', 'dispositivo'... ${ }^{31}$ ).

\section{De la metafisica a la política}

Si Heidegger concluye que la técnica no es sólo la expresión más acabada del mundo moderno sino, más grave aún, el horizonte último de comprensión de la totalidad de nuestra experiencia actual, puede decirse entonces que el problema de la técnica se sitúa en el centro de su

30 "La question de la technique", Essais et conférences, op. cit., p. 20.

${ }^{31}$ George Steiner, 1986, México, FCE, p. 182. 


\section{JUAN CRISTÓBAL CRUZ}

reflexión filosófica. Esto explica sus derivaciones políticas en 1935. Creyendo que lo político debe subordinarse (y ireducirse!) a lo metafisico, Heidegger interpreta el 'gigantismo americano' y el 'comunismo científico' como diferentes expresiones del mismo fenómeno de fondo: la era de la técnica. Es decir, los proyectos políticos de la democracia y el comunismo deben ser interpretados a la luz (o debiéramos decir, bajo la misma oscuridad en la que 'todos los gatos son pardos') del mismo fenómeno de decadencia del hombre moderno y del olvido del Ser. ${ }^{32}$

La única alternativa y la única esperanza metafisica de nuestra época ${ }^{33}$ cree encontrarlas en el movimiento nacionalsocialista y en la Alemania de Hitler. En 1934, Heidegger escribe a propósito de Hölderlin, 'poeta que anuncia el futuro de los alemanes', “... (su) obra (es)... el comienzo de otra historia, esta historia que se abre con el combate en el cual se decide la huida o la llegada de un dios". "Sin embargo, rápidamente, después de su fracaso político y moral como rector de la Universidad de Friburgo, cuando había tomado una posición cercana al nazismo, éste será interpretado como una expresión más del mundo de la Técnica. ${ }^{35}$

${ }^{32}$ Ver Nicolas Tertulian, "Histoire de l'Etre et révolution politique, réflexion sur un ouvrage posthume de Heidegger", Temps modernes, $\mathrm{n}^{\circ} 523$, febrero 1990, p. 120-9.

${ }^{33}$ Según sus propias palabras en entrevista a Rudolf Augstein y Georg Wolff, Respuestas y preguntas sobre la historia y la política, Der Spiegel, publicada póstumamente el 31 de mayo de 1976.

${ }^{34}$ Heidegger, Los himnos de Hölderlin, la Germania y el Rin, 1988 (1944), París, Gallimard, p. 12.

${ }^{35}$ Jacques Derrida sugiere que las ilusiones políticas del rectorado se explican por motivos filosóficos: se trata de una recaída en una comprensión de tipo metafísico de la cual Heidegger trataría de liberarse ulteriormente. En una hipótesis más convincente, Habermas sostiene que el giro de la filosofía de Heidegger se debe, en lo fundamental, a su fracaso político ( $D e$ l'esprit, 1987, París, Galilée). No deja de ser sorprendente tanto el homenaje de la gran pensadora política $H$. Arendt a Heidegger, en ocasión de sus ochenta años, como que Derrida se haya sentido obligado a defender (filosóficamente) la posición política de Heidegger. 
ROBERT MUSIL

Este deslinde político, implícito en su nueva lectura filosófica, tiene poco valor histórico y moral, pues sólo es tratado en sus seminarios y prudentemente será publicado después de la guerra (además se trata de una discusión limitada al interior del nazismo, al señalar que el movimiento habría perdido 'su justa orientación').

Luego de su fracaso político, ${ }^{36}$ Heidegger abandona la creencia en la capacidad del Dasein para influir activamente sobre el destino de la técnica, por lo que, de ahora en adelante, verá como ilusorias todas las posiciones políticas, incluyendo la democracia y el Estado de derecho. ${ }^{37}$ Así, en La cuestión de la técnica (1954), la técnica como dispositivo se convierte en "el modo según el cual lo real se devela como fondo". "Preguntamos aún: ¿esta desocultación tiene lugar más allá de todo acto humano? No. Pero no tiene tampoco lugar sólo en el hombre, ni a través de él de una manera determinante. ${ }^{238}$ Esta declaración evidencia que el pensamiento de Heidegger sucumbe a un fuerte escepticismo de la acción (política) y se refugia en la espera indefinida de 'un nuevo comienzo' que haga posible volver a encontrar, como él lo declara, 'la verdad del Ser'.

Las conclusiones que Heidegger deriva de su concepción de la ciencia y la tecnología de pensar la ciencia desde la técnica y olvidar las diversas formas de investigar en las ciencias y los muchos usos de la técnica, como dice Carlos Pereda, ${ }^{39}$ serán en extremo problemáticas. Así, en 1949 cree poder reducir a Auschwitz en su única referencia a este terrible hecho, a un mero 'accidente' de la lógica tecnológica de la modernidad: "la agricultura es ahora una industria motorizada, en cuanto a su esencia la misma cosa que la fabricación de cadáveres

${ }^{36}$ En su libro L'ontologie politique de Martin Heidegger (1988, París, Minuit), Pierre Bourdieu muestra de manera bastante convincente, cómo la evolución del pensamiento del autor es condicionada por sus posicionamientos políticos y sociales.

37 Véase la entrevista con Der Spiegel, op. cit.

38 "La question de la technique" en Essais et conférences, op. cit., p. 32. ${ }^{39}$ Carlos Pereda, "Peligro de contaminación: cierto Heidegger", Razón e incertidumbre, 1994, México, Siglo XXI, p. 211. 


\section{JUAN CRISTÓBAL CRUZ}

en las cámaras de gas y los campos de exterminación...". 40 Al considerar a la técnica como una entidad que actúa con móviles propios e independientes ${ }^{41}$ Heidegger no sólo cae en lo que Dewey (1948) llama una 'mitología teórica animista' sino en una relativización de los hechos ('Auschwitz no es más que otro evento propio de la modernidad') y su desresponsabilización - puesto que se trata 'en realidad' de una más de las 'desocultaciones del Ser'- a los individuos, las formas de vida y la ideología que hicieron posible ese crimen contra la humanidad.

Como veremos a continuación, si bien la interpretación de Heidegger es sugestiva, Musil ofrece una visión alternativa que sortea las dificultades intelectuales, históricas y morales del anterior.

\section{La olvidada magia de las ciencias}

Cuando Musil escribe 'el hombre alemán como síntoma' (Der deutsche Mensch als Symptom, 1923), los tópicos de la crítica que Heidegger desarrollará más tarde, no sólo son frecuentes sino que forman parte de la matriz del Zeitgeist dominante. ${ }^{42}$ Ya en ese ensayo, Musil protestará

42 contra ese modo categórico y unívoco de concebir a la ciencia y a la modernidad:

Se trata ahí únicamente de explicaciones negativas, como lo son en substancia los remedios preconizados... Se han derramado torrentes de lágrimas sobre la pérdida de nuestra alma, nuestra mecanización, nuestro espíritu de cálculo, nuestra irreligión; las realizaciones del arte, como las de la ciencia, son consideradas como tristes excesos de este estado de cosas... Todo el mundo, con excepción de los socialistas, buscan la salud en una regresión, en un rechazo del presente. ${ }^{43}$

${ }^{40}$ Conferencia pronunciada en Breme en 1949.

${ }^{41}$ John Dewey, La reconstrucción de la filosofia, 1993, España, Planeta, p. 28.

${ }^{42}$ Ver Pierre Bourdieu, op. cit.

${ }^{43}$ Musil, G. W. II, p. 362. 
ROBERT MUSIL

La interpretación del valor cultural y social de las ciencias defendido no se reduce entonces a un simple juicio perentorio. Musil es plenamente consciente que al nivel de la ciencia no es posible resolver los problemas fundamentales del ser humano, al igual que no desconoce el lado perverso, la frialdad, el apetito calculador y la obsesión por los hechos que dominan a la labor científica. Pero su propia experiencia en la ciencia y su interés en seguir de cerca sus debates le permiten una visión y un juicio muy distintos de los que procedían del campo literario y filosófico centroeuropeo. Su formación lo lleva a reconocer la innegable capacidad de la ciencia para mostrar posibilidades de pensamiento y soluciones un día antes insospechadas; su fuerza intelectual hace insostenibles las viejas representaciones metafísicas y morales respecto de la misma -como subraya J. Bouveresse. ${ }^{44}$ Esto explica por qué, en la antípoda del prejuicio -que aún en esos días fomenta la anacrónica concepción mecanicista- de que la ciencia desencanta el mundo, Musil afirmaba que "toda la audacia intelectual reside hoy en día en las ciencias exactas. No es en Goethe, Hebbel, Hölderlin de quienes aprendemos, sino de Mach, Lorenz, Einstein, Minkowski, Couturat, Russell, Peano..." ${ }^{45}$ Esta fascinación ante la capacidad subversiva de la ciencia es una de las constantes en Musil desde su primera gran obra, Las tribulaciones del joven Törless, donde muestra la poderosa atracción y el vértigo que ejerce la ciencia sobre quien se interese en los problemas fundamentales del ser humano. Es el sentimiento del joven cuando con las matemáticas, y en particular con los números imaginarios, es iniciado al saber de que el mayor grado de exactitud del conocimiento se acompaña de la mayor indeterminación; es decir, Törless entiende que la frontera entre la conciencia diurna y la nocturna es frágil y porosa.

Siguiendo esta línea que bajo la influencia de Mach interesa particularmente a Musil sostendrá que las matemáticas hacen posible un

44 Jacques Bouveresse, La science sourit dans sa barbe en Robert Musil, 1990, Francia, L'arc, p. 29.

${ }^{45}$ Musil citado por Bouveresse, ibid., p. 11. 


\section{JUAN CRISTÓBAL CRUZ}

máximo de economía del pensamiento, al permitir operaciones sin ella impensables: ${ }^{46}$ "hoy en día un simple asistente es capaz de realizar rápidamente problemas que su profesor, hace doscientos años solamente, no habría podido resolver sin consultar a Newton en Londres o a Leibniz en Hannover". ${ }^{47}$ No sólo es claro que la teórica del conocimiento ha realizado un progreso evidente en las matemáticas, también con ella se han conseguido indudables beneficios en la vida cotidiana, aunque su aprovechamiento técnico resulte bastante limitado: "El hombre medio no utiliza mucho más de lo que se le enseñó en la escuela primaria, el ingeniero, apenas lo necesario para orientarse en las columnas de fórmulas de los manuales técnicos, es decir poca cosa; el mismo físico, ordinariamente, trabaja con medios matemáticos relativamente poco diferenciados." 48 Para Musil, la civilización muestra las innumerables ventajas (en el sentido de economía de la actividad humana) derivadas de las aplicaciones del conocimiento matemático.

Por otra parte, considera exagerada la acusación de subordinación a la aplicación técnica de la ciencia y del científico: el auténtico matemático, defiende, tiene como única finalidad las matemáticas mismas: "Sin duda piensa que su trabajo terminará por aportar algún día una ventaja explotable, pero no es eso lo que lo estimula; él está al servicio de la verdad, es decir de su propio destino, y no del fin de este destino. ${ }^{\prime 49}$ Por lo mismo, la imagen del científico como un ser frío y

${ }^{46}$ Jean-Pierre Cometti, Robert Musil ou l'alternative romanesque, 1985, París, PUF, p. 29. La noción de economía del pensamiento (Denkoekonomie) era fundamental para Mach: "Cuando es posible (gracias a la ciencia), caracteres comunes a varios fenómenos son puestos en relieve de una vez y para siempre": la descripción simbólica científica debe ser, al mismo tiempo, lo más simple y completa posible (véase Xavier Verley, Mach, 1988, París, PUF); esta noción de economía del pensamiento remonta al nominalismo de Guillermo de Occam.

${ }^{47}$ Gesammelte Werke II, op. cit., p. 56.

${ }^{48}$ Ibid., p. 57.

${ }^{49}$ Musil, L'homme mathématique, Essais, 1978, París, Seuil, p. 58. (Der mathermatische Mensch (1913), G. W., II, op. cit., p. 1006.) 
ROBERT MUSIL

desapasionado tampoco le parece verdadera, al contrario, éste debe ser considerado como una de las pocas sobrevivencias de una vida motivada: "Fuera un milagro de economía, el resultado práctico de su actividad, lo que lo posee, es la prodigalidad y la pasión."

Desde esta óptica, al contrario de lo que parece a primera vista y defiende Heidegger en $E l$ ser y el tiempo, ${ }^{51}$ Musil sostiene que las operaciones de un científico, como el matemático, no son mera prolongación de la astucia. Aunque nos aprovechemos de ellas en la vida cotidiana, derivan de un ámbito más vasto:

Ahora bien, cuando todo ello ha tomado la más bella forma del mundo, he aquí que los matemáticos (infatigables entrometidos teóricos) descubren de golpe, en el fundamento mismo de la elaboración, algún vicio irremediable: y constatan, yendo al fondo de las cosas, que ¡la construcción entera se sostiene sobre el vacío! Pero las máquinas funcionan. ${ }^{52}$

Pese a lo que defiende Heidegger, la ciencia no reduce al ente a la categoría de objeto manipulable ni se limita a desplazarse sobre terreno conocido, ${ }^{53}$ parece más bien una de las experiencias más cercanas al misterio, que equivale a reconocerle una cercanía al mal, como en Törless y U1rich, debido a su capacidad de negación de toda certeza. No es extraño entonces que por la vía de las matemáticas, Musil se aproxime a la concepción de la verdad de Nietzsche: "Nos vemos entonces reducidos a concebir que nuestra existencia es fantasmagoría pura; la vivimos, cierto, pero solamente ;en virtud de un error sin el cual ella no sería! No hay ningún hombre, hoy en día, que experimente lo fantástico más cerca que el matemático." ${ }^{94}$

${ }^{50}$ Musil, ibid., p. 57.

${ }^{51}$ Respecto a este punto, véase Hubert Dreyfus, op. cit., p. 174.

${ }^{52}$ Musil, ibid., p. 58.

${ }^{53}$ Heidegger, Nietzsche, t. I, op. cit., p. 372. (Nietzsche, Neske, 1969, p. 494 .)

${ }^{54}$ Musil, ibid., p. 58. 


\section{JUAN CRISTÓBAL CRUZ}

Todo lo alejaba de la oposición neo-romántica entre la ciencia y el sentimiento; la ciencia y el pensamiento reflexivo en general deben ser considerados como la condición necesaria para restituirle un alma al mundo moderno. Como subraya Jean Pierre Cometti, ${ }^{55}$ sólo así es comprensible que el enorme interés de Musil por Mach lo lleve, a encontrar una vía para desarrollar las implicaciones de la ciencia para la vida ${ }^{56}$ Musil no puede compartir la tajante división que defienden entonces el primer Wittgenstein y Carnap, según la cual a diferencia de las éticas o estéticas, sólo las proposiciones científicas poseen significación. Tampoco pretende reducir los filosóficos a problemas científicos. Musil se encuentra en realidad más cerca del joven Popper, ${ }^{57}$ quien defiende la necesidad de distinguir las diversas formas de experiencia sin por ello pensar que las proposiciones 'metafísicas' estarían desprovistas de significación ni que no sería posible establecer formas de comunicación entre las ciencias exactas y otras formas de conocimiento.

La importancia de la permanente subversión de las formas habituales de pensar que caracteriza la labor de la ciencia, reside también en que implica aceptar las siguientes actitudes morales complementarias: en primer lugar, una voluntad de verdad capaz de asumir el valor provisorio e hipotético de las respuestas ofrecidas; en segundo, una actividad crítica constante y por ende el rechazo de las respuestas

55 Jean-Pierre Cometti, op. cit.

${ }^{56}$ Ensayos, op. cit. Se puede encontrar un interés semejante en la relación entre ciencia y sensibilidad, ciencia e intelecto, más o menos contemporáneo en Paul Valéry: "...(les sciences) nous ont refait una manière de voir" y luego "Le même individu qui peut penser physique ou biologie avec des instruments de pensée comparables ... des instruments de précision, pense politique au moyen de termes impurs, de notions variables, de métaphores illusoires", Regards sur le monde actuel, 1945, París, Gallimard, p. 17. También, L'idée fixe, Oeuvres, 1977, (1932), París, La Pléide-Gallimard.

${ }^{57}$ A este respecto ver el artículo de Popper The Demarcation between Science and Metaphysic en el cual aclara sus diferencias teóricas con Carnap y el primer Wittgenstein, durante su período vienés (alrededor de 1932). 
ROBERT MUSIL

categóricas; finalmente, una actitud de desconfianza ante las visiones sistemáticas y totalizadoras. ${ }^{58}$ Es comprensible entonces el malestar que produce en Musil la tendencia en la filosofía y en los filósofos a confeccionar respuestas globales y a pronunciarlas con el aire profético y el tono violento de los dictadores: "Probablemente ésta es también la razón por la cual las épocas de tiranía han visto surgir grandes figuras de filósofos, en tanto que las épocas democráticas y de alta civilización no logran producir un solo filósofo de valor, al menos si nos atenemos a las quejas que se escuchan comúnmente a este respecto." ${ }^{.59}$ En este sentido, Musil es plenamente consciente de que su visión y defensa de la investigación científica van a la par con las de una sociedad abierta. ${ }^{60}$

Es posible que Hilary Putnam ${ }^{61}$ no se equivoque cuando afirma que Dewey es el único filósofo defensor de la idea de investigación científica a la investigación ética, pero se equivocaría si creyese que ha sido el único en hacerlo. No sólo el autor de El hombre sin atributos es consciente, como Dewey, de que la ciencia moderna ha mostrado que el conocimiento reposa no en el estudio de las cualidades de un ilusorio objeto inmutable sino en las relaciones que participa el 'objeto'. También es claro que el proyecto ético, el estilo literario y el hecho de que a través de la técnica el personaje central de su gran novela, el ingeniero Ulrich (quizá con base autobiográfica) intente convertirse en un hombre excepcional, ${ }^{62}$ sólo se pueden entender bajo la idea de que "la inteligencia científica con su conciencia estricta, su ausencia de prejuicios y su voluntad de poner cada nuevo resultado en duda, ubica en una zona de interés de segundo plano lo que deberíamos hacer en los problemas de la vida."63

${ }^{58}$ Como es evidente, la noción de investigación implícita aquí es muy cercana a la defendida por C. S. Peirce, lo que no es sorprendente puesto que Musil conoce y lee con atención a los pensadores del pragmatismo.

${ }^{59}$ Der Mann ohne Eigenschaften, op. cit., vol. I, p. 253.

${ }^{60}$ Por ejemplo, ibid., p. 1887.

${ }^{61}$ Hilary Putnam, Définition, 1992, París, l'éclat, p. 74.

${ }^{62}$ Der Mann ohne Eigenschaften, op. cit., vol. I, p. 38.

${ }^{63}$ Musil, Geammelte Werke, op. cit., II, p. 1011. 


\section{JUAN CRISTÓBAL CRUZ}

Desde la visión negativa difundida por los seguidores de Tolstoi en lo referente a los problemas de sentido, la ciencia "no contribuye, en definitiva, a plantear adecuadamente tales cuestiones"; 64 en realidad es la respuesta de una época que, por un parte, ha esperado demasiado de ella (con la ilusión expresa o inconsciente de que remplace a la religión) y que, al mismo tiempo, no ha sabido aplicar el rigor metodológico y la capacidad de resolución que poseen las ciencias exactas a los problemas culturales de la época. Los Ensayos y la enorme elaboración de El hombre sin atributos sólo se pueden entender como la voluntad de subvertir esa visión que se limita a refugiarse en una actitud puramente reactiva ante la ciencia y llevar a cabo el proyecto más general de aprovechar los logros culturales que la ciencia ha aportado.

${ }^{64}$ Max Weber, "La ciencia como vocación”, El politico y el científico, 1981, México, Premia, p. 76. 